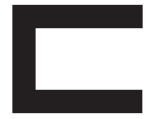

开舟正事

ER $\mathbb{R}(\mathbb{E S}$

CIENTÍFICAS

EXATAS E TECNOLÓGICAS

ISSN IMPRESSO - 2359-4934

ISSN ELETRÔNICO - 2359-4942

\title{
GERENCIAMENTO DA CADEIA DE SUPRIMENTOS - SUPPLY CHAIN MANAGEMENT A BUSCA PELA VANTAGEM COMPETITIVA
}

Suzana Arleno Souza Santos ${ }^{1}$

Alline Soares Viana²

\section{RESUMO}

Todos os passos das empresas que desejam obter vantagem competitiva estão voltados hoje para a Gestão da Cadeia de Suprimentos. Por esta razão, as mesmas têm procurado investir na implantação deste conceito. Os elementos fundamentais para o bom desempenho de um empreendimento são o planejamento e o gerenciamento do setor de suprimentos. Este trabalho tem por objetivo apresentar um entendimento mais amplo do conceito de Gestão da Cadeia de Suprimentos para que as empresas obtenham sucesso no mercado atual mostrando

o quanto é vantajosa a implementação desta ferramenta. Apresentaremos pontos importantes como a descrição da cadeia, implementação e planejamento para um bom gerenciamento, ferramentas, bem como os fatores que influenciam diretamente no sucesso da corporação.

\section{PALAVRAS-CHAVE}

Gestão. Cadeia de Suprimentos. Planejamento. Vantagem Competitiva. 


\section{ABSTRACT}

All steps of the companies that wish to gain competitive advantage are facing today for Supply Chain Management. For this reason, they are trying to invest in the implementation of this concept. The key elements to the performance of an enterprise are planning and managing the supply sector. This work aims to present a broader understanding of the concept of Supply Chain Management for companies to achieve success in today's market showing how the implementation of this tool is advantageous. Will present important points as the description of the chain, implementation and planning for good management, tools, as well as the factors that directly influence the success of the corporation.

\section{KEYWORDS}

Management. Supply Chain. Planning. Competitive Advantage.

\section{RESUMEN}

Todos los pasos de las empresas que deseen obtener una ventaja competitiva se enfrentan hoy en día para la Gestión de la Cadena de Suministro. Por esta razón, ellos están tratando de invertir en la aplicación de este concepto. Los elementos clave para el rendimiento de una empresa son la planificación y la gestión del sector de alimentación. Este trabajo tiene como objetivo presenter una comprensión más amplia del concepto de Gestión de la Cadena de Suministro para las empresas a alcanzar el éxito en el mercado actual mostrando cómo la implementación de esta herramienta es ventajosa. Presentará puntos importantes como la descripción de la cadena, la ejecución y la planificación de una Buena gestión, herramientas, así como los factores que influyen directamente en el éxito de la corporación.

\section{PALABRAS CLAVE}

Gestión. Planificación de la Cadena de Suministro. la Ventaja Competitiva 


\section{INTRODUCÃO}

A Gestão da Cadeia de Suprimentos (SCM) é um tema que está alcançando um crescente destaque no meio acadêmico e empresarial, pois se trata de um fator chave para o sucesso atual das empresas que estão dentro de um ambiente de competição, que já não é mais individual e sim entre as cadeias de suprimentos (CARVALHO, 2005).

No período compreendido entre 1950 e 2000, ocorreram grandes transformações nos conceitos de gerenciamento das organizações. Com a chegada da qualidade total nas empresas e os programas de crescimento, houve também um avanço na qualidade e na produtividade (COOPER, 1997).

O SCM propõe uma rede de facilidades e opções de distribuição para garantir a obtenção de materiais, a transformação destes em produto intermediário ou final e a distribuição destes para os consumidores. o SCM pode ser implantado tanto em organizações de manufatura quanto em prestadoras de serviços (CHRISTOPHER, 1997).

Na primeira fase, que foi até 1960, havia estoques para suprir a falta de visão de toda a cadeia. Na fase seguinte, que durou de 1960 a 1980, as atividades já estão interligadas, porém, a visão da cadeia ainda era baixa e de curto prazo. De 1980 a 1990, buscou-se atender a cadeia interna, com integração, decisões que passaram a basear-se nos históricos, e planejamentos que aumentaram de curto para médio prazo (NAZÁRIO, 2000).

Na última fase, a partir de 1990 até os dias de hoje, o grande objetivo e o maior desafio de todas as empresas, passou a ser conseguir reduzir os custos e melhorar a eficiência no atendimento ao cliente. Segundo Nazário (2000), desde então, o conceito SCM tem sido empregado nas grandes organizações para melhorar a eficiência, fazendo assim com que crescesse o nível de serviço demandado por fornecedores, consumidores, atacadistas, varejistas, operadores logísticos, clientes finais, entre outros.
0 perfeito relacionamento entre estes parceiros é o que propicia melhorias à cadeia de suprimentos. Somente com a evolução do método e o aperfeiçoamento da comunicação com os fornecedores, a empresa começa a obter os benefícios e resultados esperados, apoiando-se nas melhores práticas do mercado, pois elas direcionam a organização aos principais caminhos e os resultados positivos (BALLOU, 2001).

0 ambiente industrial, atualmente, ainda está numa profunda reflexão dos modelos, sobre o desenvolvimento dos sistemas produtivos. O fundamento da produção industrial, comercialização e de relacionamento entre empresas e pessoas está sendo revista, ocasionando grandes mudanças da cadeia de suprimentos das indústrias. Originando assim, ao Gerenciamento da Cadeia de Suprimentos (PIRES, 1998).

O Gerenciamento da Cadeia de Suprimentos (SCM) começou a se desenvolver a fim de alcançar os objetivos de eliminar redundâncias e reduzir o tempo de ciclo e inventários de forma a fornecer melhores serviços aos clientes ao menor custo (SCARVADA, 2000).

Hoje, apesar da eficácia da cadeia de suprimentos já ter sido comprovada pelos resultados de diversas empresas que a implantaram, muitas esbarram no seu custo de implantação. Faz-se necessário um grande investimento de recursos financeiros para projetar e implantar uma Cadeia de Suprimentos (COOPER, 1997).

É necessário empregar o mesmo valor para monitoramento e operação da Cadeia de Suprimentos. Um projeto de uma Cadeia de Suprimentos que seja ineficiente ou equivocado aumenta significativamente os custos pelo emprego inadequado de recursos, tomada de decisões erradas baseada em informações incorretas e danos devido ao emprego de recursos financeiros incorretos (LAMBERT, 2000). 
Embora este custo seja elevado, entende-se que a cadeia de suprimentos é o futuro de qualquer organização que queira obter vantagem competitiva de mercado. Somente com a implantação da cadeia de suprimento e um gerenciamento sem falhas, permitirá às organizações alcançarem neste ambiente tão concorrido o lucro pretendido (LAMBERT, 1998).

\section{A CADEIA DE SUPRIMENTOS}

A cadeia de suprimentos é uma metodologia criada para alinhar todas as atividades de produção, armazenamento e transporte de forma sincronizada, visando a obtenção na redução de custos, minimizar ciclos e maximizar o valor percebido pelo usuário final em busca de grandes resultados (BALLOU, 2001). Ela abrange todas as etapas envolvidas na produção e entrega de um produto final desde seu início que é o fornecedor até o cliente final.

À medida que a logística dedica-se às operações da própria empresa, a cadeia de suprimentos olha o processo desde o início até o final. Para que a cadeia atinja sua maior eficiência, faz-se necessária a total integração e colaboração entre todos os participantes (FLEURY, 2000).

Muitas são as definições para uma cadeia de suprimentos, porém, sob a perspectiva do sistema, adaptou-se o conceito de que a cadeia de suprimentos é um agrupado de cadeias de valor. A cadeia de valor é um modelo teórico que descreve como se desenvolvem as atividades de uma empresa (PORTER, 1989).

Seguindo o conceito de cadeia, esta é composta por vários elos que formam um processo econômico que começa com a matéria-prima e chega até à distribuição do produto acabado. Em cada elo, é acrescentado valor, que é, em termos competitivos, a quantia que os consumidores estão dispostos a pagar por um determinado produto ou serviço (PORTER, 1989).
A análise da cadeia de valor permite aperfeiçoar o processo produtivo, uma vez que se pode ver, detalhadamente, passo a passo, o funcionamento da empresa. 0 estudo da cadeia de valor possibilita obter uma vantagem estratégica, já que existe a possibilidade de gerar uma proposta de valor que seja única no mercado (PORTER, 1989).

Para Porter (1989), analisar a cadeia de valor de cada empresa significa avaliar todas as atividades efetuadas e a forma como interagem na organização. Alguns conceitos definem um conjunto de atividades de valor necessárias à produção e entrega de um serviço ou bem para o consumidor final. Porter (1989) originou o conceito de uma cadeia genérica de valor, que se dividem em diversas atividades que devem ser exercidas como apoio às atividades base.

A cadeia de valor representa todas as atividades que geram valor agregado para que a empresa possa atingir a demanda dos consumidores. Porter (1989) classifica as atividades de valor como primárias (operações, logística interna, marketing, logística externa, vendas e serviço) e de suporte (desenvolvimento de tecnologias, gestão de recursos humanos, aquisição de serviços e insumos e infraestrutura).

As cadeias de suprimentos operam de maneira integrada em várias partes do mundo. Uma cadeia de suprimentos é formada por uma gama de empresas consideradas estratégicas e lideradas por uma empresa líder. 0 conjunto de cadeias de valor das empresas que as compõem, caracteriza uma cadeia de suprimentos (PIRES, 2004).

Sendo assim, esse conjunto pode ser constituído por fornecedores e clientes distribuídos em todo o mundo, atendendo a necessidades mútuas. Para o autor, os objetivos da cadeia de suprimentos atravessam a diminuição dos custos de produção e aumento da agregação de valor ao produto por meio do processo de gestão que foca em toda cadeia (COOPER, 1997). 
Para Lambert e outros autores (1998) conhecer claramente e entender como a estrutura de rede da cadeia de suprimentos está configurada é fator-chave para que a cadeia alcance o sucesso esperado por todos aqueles que a implementam, esperando obter vantagem no mercado.

Fleury (2000) criou um modelo para escolher a cadeia a partir dos produtos que são oferecidos pela empresa, onde os classifica em inovadores e funcionais. Ele faz a associação das cadeias com processos eficientes aos produtos classificados como funcionais, e as cadeias com processos classificados como produtos inovadores.

Os funcionais são produzidos para suprir às necessidades básicas dos consumidores, ao passo que os produtos considerados inovadores, representam as inovações que são incorporadas a eles, fazendo da demanda imprevisível e rápida (FLEURY, 2000).

A análise da capacidade principal dos integrantes na cadeia de suprimentos mostra processos que agregam ou não agregam valor. Para Chopra (2003), as habilidades da empresa somente devem permanecer se elas forem determinantes para assegurar uma vantagem competitiva.

Para Lambert e outros autores (1998), duas características são importantes para analisar, descrever e gerenciar uma cadeia de suprimentos quanto à sua estrutura:

- Horizontal (número de níveis que ao longo da cadeia);

- Vertical (número de clientes e fornecedores em cada nível).

Os componentes de uma cadeia de suprimentos, para Lambert e outros autores (1998), podem ser classificados em primários e secundários.
Um componente primário é uma organização autônoma que desempenha uma atividade gerencial e/ ou operacional relativa aos processos de negócios que são projetados para produzir saídas específicas a um cliente ou mercado. Um membro secundário propicia os conhecimentos, recursos e utilidades entre outros, para que um integrante primário possa exercer suas funções essenciais (LAMBERT et al., 1998).

Os membros primários e secundários conectam-se por relações mantidas entre processos de negócios. Uma cadeia de suprimentos pode ser representada pelas interações que existentes entre seus membros e os seus processos de negócios. Lambert e outros autores (1998) classificaram as relações estabelecidas entre os processos-chave em: relações gerenciadas, não gerenciadas, monitoradas e com não membros.

De acordo com Christopher (1997), a resposta na cadeia de suprimentos liga-se diretamente ao atendimento rápido dos da demanda do mercado. Outro ponto muito discutido quanto à cadeia de suprimentos faz referência aos diversos tipos de relacionamentos e suas motivações.

O grau de relação que é mantido entre uma empresa focal e seus parceiros mostra o nível de integração e coordenação entre as organizações em função dos objetivos na gestão de uma cadeia. A integração passa pelo aprimoramento e comprometimento de todas as partes das parcerias. Cooper (1997) destaca seis razões para explicar a consolidação de parcerias: necessidade, assimetria, reciprocidade, eficiência, estabilidade e legitimidade.

Os participantes da cadeia de suprimentos e todos os seus negócios conectam-se entre os principais elos da cadeia. Essa conexão é consolidada num fluxo de informação, cujo nível de eficiência associa-se ao processamento das informações (COOPER, 1997).

Para Chopra e Meindl (2003), a informação representa uma ligação entre as diversas etapas da 
cadeia de suprimentos, o que permite que possam conduzir ações e por em prática os benefícios de maximizar a lucratividade total da cadeia. As conexões requerem quatro elementos: softwares, hardwares, operadores do sistema, gestores, e integração desses elementos no fluxo das informações na cadeia de suprimentos.

A tecnologia da informação deve ser utilizada como recurso necessário ao processo de união e conexão da cadeia colabora para a consolidação dos procedimentos de comunicação (CHOPRA, 2003).

\section{FERRAMENTAS APLICADAS NO GERENCIAMENTO DA CADEIA DE SUPRIMENTOS}

Embora grandes vantagens tenham sido alcançadas com a implantação da cadeia de suprimentos, poucas empresas implementam corretamente ou utilizam seu conceito. As razões são principalmente duas, o conceito que é relativamente novo e pouco disseminado entre os profissionais e a complexidade e dificuldade de implementação do conceito, que causa diversas mudanças nas ações internas e externas da empresa (FLEURy, 2000).

Em geral, as grandes empresas possuem tecnologia para viabilizar a cadeia, mas normalmente esses recursos tecnológicos são utilizados de forma incorreta. Para uma correta implantação da cadeia de suprimentos podem ser utilizadas diversas ferramentas, cada uma com uma finalidade individual que pode ser utilizada para dar rapidez no fluxo interno e externo de informações, apoiar as decisões e melhorar a eficácia do planejamento de aprovisionamento, produção e vendas (BALLOU, 2001).

Apesar de existirem inúmeras alternativas, apresentaremos ferramentas que são consideradas mais importantes no processo de implantação e integração da cadeia de suprimentos (LAMBERT, 1998).

\subsection{DIAGRAMA DE FLUXO DE PROCESSOS}

Uma etapa fundamental para o processo de implantação do SCM em qualquer empresa é a análise de sua cadeia de suprimentos para identificar com clareza os gargalos e operações que não agregam algum tipo de valor para o produto ou cliente final (SCRIBD, s.d.).

O Diagrama de Fluxo de Processo é uma ferramenta de análise que detalha por meio de determinados símbolos cada etapa e operação, fazendo a análise e detathamento de todas as interfaces com as áreas de apoio como, recursos humanos, qualidade, compras, vendas, assistência técnica, entre outras (SCRIBD, s.d.).

O Diagrama que pode ser visto na figura abaixo é regularmente criado em workshop e conta com a participação de representantes de todas as áreas para possibilitar o detalhamento de todas as etapas relacionadas com o fluxo de produto ou fluxo de informações dentro da cadeia (UNIVERSIDADE CÂNDIDO MENDES).

Figura 3.1 - Exemplo de Fluxo de Processos

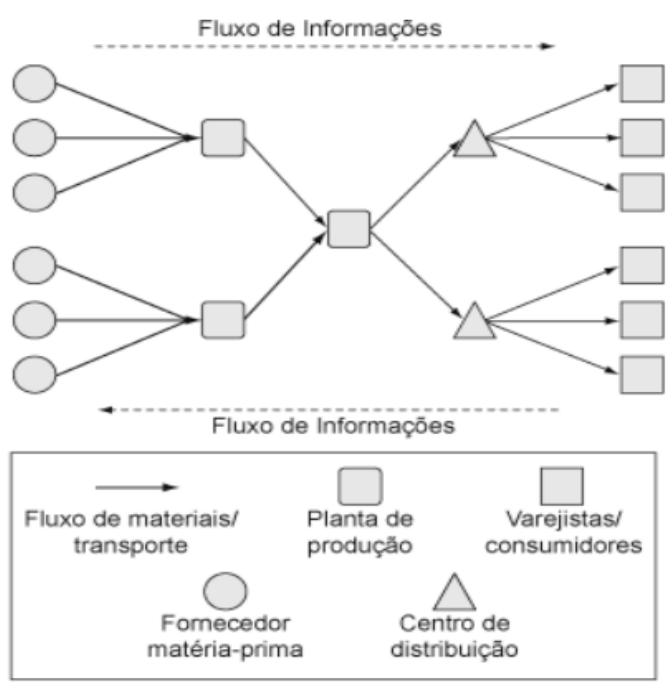

Fonte: www.google.com.br/imagens. Acesso em: 13/4/2013. 
A utilização dessa ferramenta facilita o entendimento do processo, ajuda a identificar as oportunidades de melhoria, ou seja, identificar os gargalos e as operações que não agregam nenhum tipo de valor para o cliente, e auxilia no desenvolvimento de produtos e serviços (UNIVERSIDADE CÂNDIDO MENDES).

\subsection{APS - ADVANCED PLANNING SCHEDULING}

O Advanced Planning Scheduling consiste em softwares de otimização de toda a cadeia de suprimentos, que agrega desde o planejamento da demanda, produção e distribuição o que possibilita conectar as decisões na cadeia de suprimento a fim de administrá-las de maneira integrada (Kurbel. et. al., 2014)

O APS é uma tecnologia bastante recente baseado na Teoria das Restrições (TOC). Este método de administração da produção foi desenvolvido com a finalidade de solucionar problemas que estão relacionados à logística. Possibilita fazer todo o planejamento de uma fábrica, como por exemplo, definir quais materiais serão comprados, produzidos e em que sequência esse processo deverá seguir. (SCHNEIDER ELECTRIC).
A eficácia do APS depende diretamente da rapidez e qualidade, e do fluxo das informações. Um dos sistemas que pode garantir a qualidade e rapidez desse fluxo é o Enterprise Resources Planning (ERP) que consiste em um banco de dados que permite a troca das informações internamente na empresa, o que possibilita que a empresa trabalhe com dados unificados. É imprescindível que a organização possua total controle de seus processos (Kurbel. et. al., 2014)

O APS representado na Figura 3.1 provoca na empresa a redução de custos, permitindo que o lucro alcançado seja investido na melhoria do processo. Os ganhos são grandes, dependendo do nível de controle da empresa. O nível de integração em que a empresa se encontra e a qualidade de seu banco de dados, determinarão a necessidade de apenas alguns módulos ou do sistema completo. O APS é crucial para implantação do Supply Chain Management (AVANÇADO DE PROCESSO COMBINATÓRIA, INC.).

Figura 3.2 - Exemplo de APS

O algoritmo de TOC submete todo estudo aos recursos que a fábrica possui e de toda sua linha de produção, gerando muitos benefícios. O mais importante é a redução dos estoques intermediários o que gera menos dinheiro investido em forma de inventário e também auxilia no cumprimento dos prazos das entregas dos produtos aos clientes, aumentando significativamente o nível e a qualidade do serviço que é prestado (SCHNEIDER ELECTRIC).

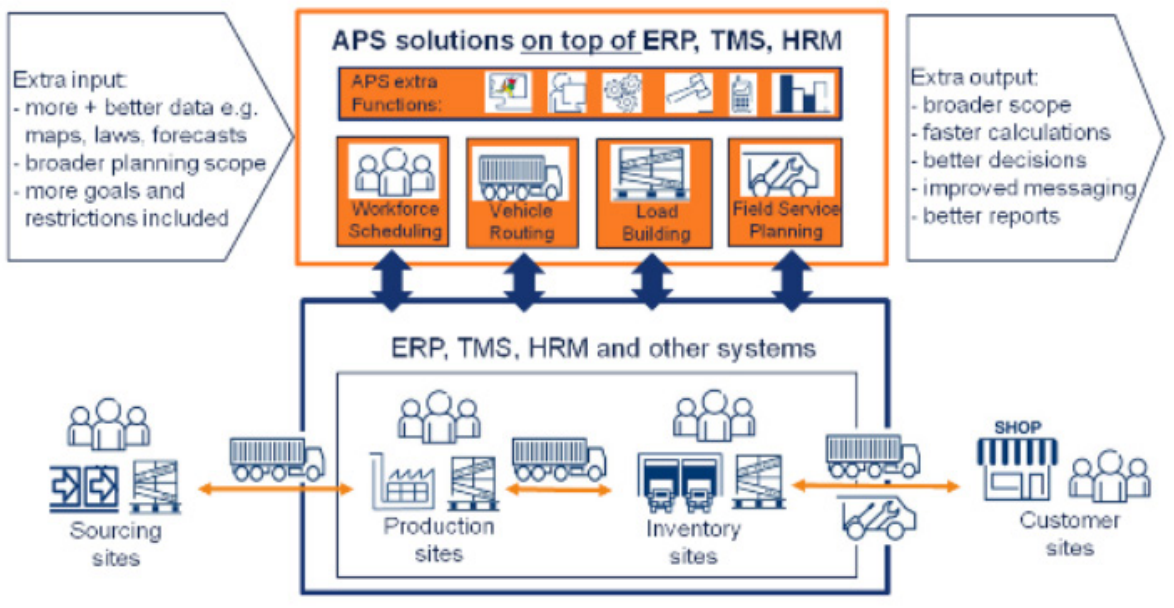

Fonte: www.google.com.br/imagens. Acesso em: 13/04/2013. 


\subsection{CPFR - COLLABORATIVE PLANNING, FORECASTING AND REPLENISHMENT}

O Planejamento Colaborativo, Previsão e Reabastecimento (CPFR) é a mais recente iniciativa para obter ganhos por meio do melhor gerenciamento do fluxo de informações ao longo das cadeias de suprimento, porém foca especificamente no planejamento de colaboração entre as empresas que participam diretamente da cadeia de suprimentos (ZUPPI, 2009).

Não pode ser considerado como um programa de resposta rápida, pois os programas de resposta rápida têm o objetivo de permitir que os fabricantes e os clientes reajam rapidamente de acordo com a demanda do consumidor final, reduzindo o estoque existente em toda a cadeia de suprimento e aumentando a disponibilidade do produto (AROZO, 2000).

Busca, também, redução nos níveis de estoque e melhoria nos níveis de serviço. É baseado na elaboração conjunta das previsões de venda e no planejamento de suprimento, levando em conta os limites que existem na cadeia de suprimentos, tanto do fornecedor, varejista ou distribuidor. É um modelo de gestão da cadeia de suprimento altamente vantajoso, tanto para os varejistas quanto para os fabricantes (VIVALDINI; SOUZA, 2006).

É definido como um conjunto de normas e procedimentos sustentado pelo Voluntary Interindustry Commerce Standards (VICS), um comitê que foi fundado e formado por representantes de várias organizações, objetivando o aumento da eficiência das cadeias de suprimentos (SCCORI, s.d.).

Para os fornecedores, o CPFR representa a real oportunidade de alcançar crescimento nas vendas, decrescimento dos níveis de estoque e dos ciclos operacionais. Isso ocorre, pois existe a possibilidade de realizar o planejamento da produção e a distribuição da demanda final, possibilitando a realização de ações em conjunto com o varejista (GOMES; MANCINI, 2014).
Este modelo de gestão impacta profundamente na gestão da cadeia de suprimentos, gerando diversos benefícios. Entre eles, aumento nas vendas dos produtos, redução no erro de previsão, redução nos níveis de estoque com consequente aumento no giro dos mesmos, aumento da disponibilidade de produto para os varejistas, aumentos no nível de serviço dos fornecedores, redução dos custos de planejamento de produção conforme Figura 3.3 (GOMES; MANClNI, 2014).

Figura 3.3 - Exemplo de CPFR

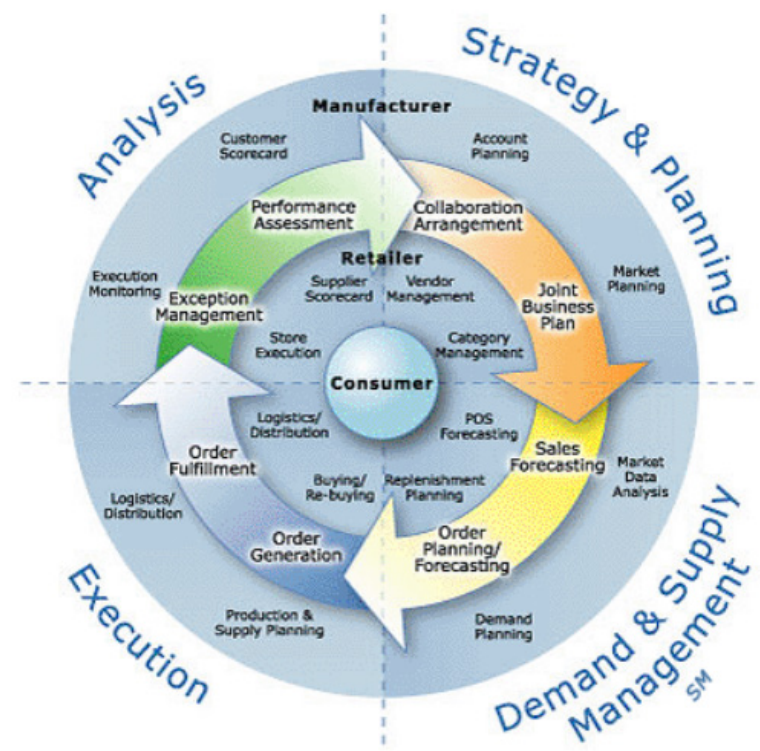

Fonte: www.google.com.br/imagens. Acesso em: 13/4/2013.

\subsection{SUPPLY CHAIN MANAGEMENT - SCM}

A gestão de cadeia de suprimentos (Supply Chain Management) permite melhorar e desenvolver as relações entre cada uma das organizações envolvidas em uma cadeia de suprimentos. Integra os fluxos de materiais e informação para realizar o trabalho de maneira otimizada, ao mesmo tempo em que reduz custos (BALLOU, 2001). 
Para realizar a gestão da cadeia de suprimentos deverá ter todas as atividades de gestão e logística, presentes em cada fase do processo. As cadeias de suprimento são mais complexas em um contexto mais globalizado onde os consumidores são cada vez mais exigentes e as decisões mudam continuamente, assim como na economia. A eficiência do supply chain management não somente é indispensável para o sucesso como é uma condição primordial para a sobrevivência da empresa (COOPER, 2000).

De acordo com Pires (1998), uma gestão na cadeia de suprimentos otimizada será a principal solução para aumentar a rentabilidade de sua empresa. Sendo uma ferramenta empresarial imprescindível, esta solução ajudará a alcançar os objetivos relacionados com as metas pessoais da empresa.

O Supply Chain Management observado na Figura 3.4 abaixo, lhe permite entregar o produto a tempo de forma e qualidade a satisfazer as necessidades de seus clientes, realizar um balanço adequado para tomar melhores decisões, promover um serviço de excelência ao consumidor final, contar com a capacidade de entrega da variedade de produtos que a empresa ofereça (PIRES, 1998).

\section{Figura 3.4 - Exemplo de SCM}

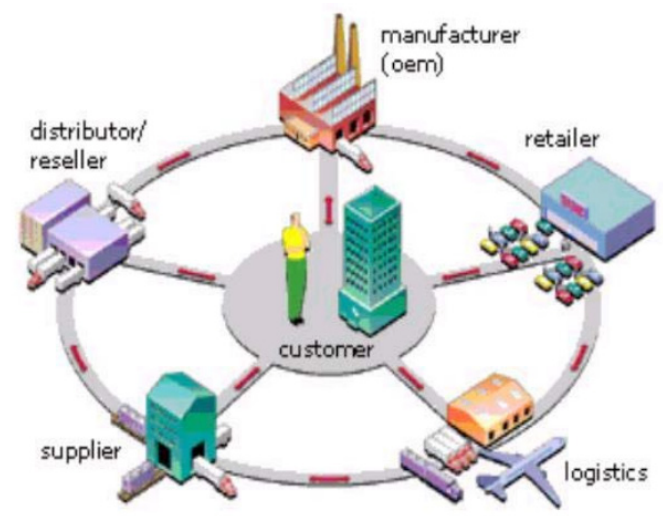

Fonte: www.google.com.br/imagens. Acesso em: 13/4/2013.

\section{CONCLUSÃO}

A competitividade global no qual o ambiente empresarial está envolvido tem levado as empresas a buscarem mudanças tanto na sua estruturação interna, o que diz respeito a programas e técnicas de melhoria e redução de custos, quanto na sua estruturação com o meio externo, o relacionamento com fornecedores e clientes.

A utilização crescente da tecnologia da informação e as modernas ferramentas de SCM, são essenciais para aumentar a competitividade entre as empresas proporcionando a otimização dos recursos disponíveis, reduzindo assim os custos empregados no processo e permitindo consequentemente $\mathrm{o}$ aumento dos lucros.

Porém, ainda são muitas as dificuldades enfrentadas na implementação do SCM. Ainda que hoje seja comprovado que o gerenciamento da cadeia de suprimentos propicia redução dos custos, qualidade, flexibilidade e melhor atendimento ao cliente, as empresas ainda esbarram em algumas dificuldades para o implementar.

Mesmo hoje, quando há uma consciência maior dos benefícios trazidos pelo SCM, outras dificuldades são enfrentadas. Uma delas é o risco de em decorrência do maior fluxo de vazar informações sigilosas para concorrentes e o risco de empresas menores se tornarem dependentes das maiores por não poderem negociar com outras empresas não pertencentes à cadeia de suprimentos.

Outro fator é a dificuldade de administrar o relacionamento que deve existir entre os diferentes elos da cadeia, pois nem sempre todos os são igualmente interessados e compromissados. Muitas empresas não entendem o conceito de SCM e por muitas vezes o adotam de forma incorreta e creditam que a aquisição de um software será a solução de todos os problemas da organização. 
Cabe ressaltar que o SCM não se resume a um software, e sim envolve um amplo e complicado conjunto de fatores que requer uma profunda modificação na cultura de cada empresa. Cultura esta, que até o momento, é representada pela busca de ganhos individuais e não na busca de ganhos a partir da cadeia de suprimentos como um todo.

\section{REFERÊNCIAS}

AVANÇADO de Processo Combinatória, Inc. Programação, otimização, planejamento de capacidade. Disponível em: <http://www.combination.com/>. Acesso em: 27 abr. 2013.

BALLOU, R.H. Gerenciamento da cadeia de suprimentos: Planejamento, organização e logística empresarial. Porto Alegre: Bookman, 2001.

CARVALHO, Marcius Fabius. Importância da Informação no Desempenho da Cadeia de Suprimentos, 2005.

CHOPRA, Sunil; MEINDL, Peter. Gerenciamento da Cadeia de Suprimentos: estratégia, planejamento e operação. São Paulo: Prentice Hall, 2003.

CHRISTOPHER, M. Logística e Gerenciamento da Cadeia de Suprimentos: Estratégias para a redução de custos e melhoria dos serviços. São Paulo: Pioneira, 1997.

COOPER, M.C ; LAMBERT,D.; PAGH,J. SMC-more than a new name for logistics. International Journal of Logistics Management, 1997.

CPFR - Collaborative Planning, Forecasting, and Replenishment. Disponível em: <http://www.sccori. com/SCM/COLLABORATIVEPLANNINGFORECASTING.pdf>. Acesso em: 27 abr. 2013

FLEURY, Paulo Fernando. Supply Chain Management: Conceito, Oportunidades e Desafios da Implementação, 2000.
GOMES, Gabriela Pereira; MANCINI, Mônica Caroline. Planejamento tributário em uma empresa no ramo do comércio. 2014. Trabalho de Conclusão (Curso de Ciências Contábeis). Faculdade Sul Brasil-FASUL. Toledo-PR, 2014. Disponível em: <https://www.fasul. edu.br/publicacoes-online/app/webroot/files/trabalhos/20141203-221858.pdf>. Acesso em: 27 abr. 2014.

Kurbel, Karl; Becker, Jörg; Gronau, Norbert; Sinz, Elmar; Sushi, Leena. Enzyklopãdie Der Wirtschaftsinformatik. Potsdam, 2014. DIsponível em <http://www. enzyklopaedie-der-wirtschaftsinformatik.de> Acesso em: 27 abr. 2014

LAMBERT, Douglas M.; COOPER, Martha C. Issues n Supply Chain Management. Industrial Marketing Management, 2000.

LAMBERT, D.M ; COOPER, MC; PAGH,J.D. Supply Chain Management: Implementation issues and research opportunities. The International Journal of Logistics Management, 1998.

NAZÁRIO, Paulo. A Importância de Sistemas de Informação para a Competitividade Logística, 2000.

PIRES, S. R. Gestão da cadeia de suprimentos (Supply Chain Management): Conceitos, estratégias, práticas e casos. São Paulo: Atlas, 2004.

PIRES, S. R. I. Managerial implications of the modular consortium model in a Brazilian automotive plant. International Journal of Operations \& Production Management, 1998.

PORTER, Michael. Competitiva Estrategy. New York: Free Press, 1989.

SCAVARDA, Luiz Felipe \& HAMACHER, Sílvio. A Evolução da Cadeia de Suprimentos, 2000.

SCRIBD. Diagrama de Fluxo de Processo. Disponível em: <http://pt.scribd.com/doc/92112762/5-Dia- 
grama-de-Fluxo-de-Processo>. Acesso em: 27 abr. 2013.

SOUZA, Paulo Teixeira de. Logística interna para empresas prestadoras de serviço, 2002.

SCHNEIDER ELECTRIC. Solvelt Software está agora Schneider Electric. Disponivel em: <http://www2. schneider-electric.com/sites/corporate/en/products-services/former-brands/solveit/solveitsoftware. page >. Acesso em: 27 abr. 2013.

SCCORI. Materiais de Leitura Curso de Gestão de Cadeia de Suprimentos. Disponível em: <www.sccori. com>. Acesso em: 27 abr. 2013.

UNIVERSIDADE CÂNDIDO MENDES. Diagrama de Fluxo de Processo. Disponível em: <http://online. ipanema.ucam.edu.br/alunos/pdfs/osm_08.pdf $>$. Acesso em: 27 abr. 2013.
VIVALDINI, Mauro; SOUZA, Fernando Bernardi de. Implantação de um CPFR (Collaborative Planning, Forecasting, and Replenishment) por intermédio do prestador de serviços logísticos (PSL). Anais do IX Simpósio de Administração da Produção, Logística e Operações Internacionais - SIMPOI, FGV-EAESP, 2006. Disponível em: <https://www. fasul.edu.br/publicacoes-online/app/webroot/ files/trabalhos/20141203-221858.pdf>. Acesso em: 27 abr. 2013.

ZUPPI, Ana Claudia. Collaborative Planning, Forecasting and Replenishment (CPFR), 4 de setembro de2009. Disponível em: <http://www. administradores.com.br/artigos/tecnologia/collaborative-planning-forecasting-and-replenishment-cpfr/33456/>. Acesso em: 27 abr. 2013. 